Man and Nature

L'homme et la nature

\title{
La Philosophie politique de Kant
}

James Crombie

Volume 1, 1982

URI : https://id.erudit.org/iderudit/1011793ar

DOI : https://doi.org/10.7202/1011793ar

Aller au sommaire du numéro

Éditeur(s)

Canadian Society for Eighteenth-Century Studies / Société canadienne d'étude du dix-huitième siècle

ISSN

0824-3298 (imprimé)

1927-8810 (numérique)

Découvrir la revue

Citer cet article

Crombie, J. (1982). La Philosophie politique de Kant. Man and Nature / L'homme et la nature, 1, 81-92. https://doi.org/10.7202/1011793ar

Copyright (c) Canadian Society for Eighteenth-Century Studies / Société canadienne d'étude du dix-huitième siècle, 1982
Ce document est protégé par la loi sur le droit d'auteur. L'utilisation des services d'Érudit (y compris la reproduction) est assujettie à sa politique d'utilisation que vous pouvez consulter en ligne.

https://apropos.erudit.org/fr/usagers/politique-dutilisation/ 


\section{La Philosophie politique de Kant}

La philosophie politique de Kant a été pendant longtemps méconnue des commentateurs, surtout de ceux qui se sont occupés de l'aspect systématique de sa pensée. Les raisons de cet état de choses sont multiples. Non la moindre de ces raisons est sans doute que Kant n'est venu que tard dans sa vie à l'élaboration et à l'expression sous forme écrite d'une théorie politique selon les principes de sa philosophie. Il a par conséquent relativement peu écrit sur des sujets relevant directement de la philosophie politique. En effet, selon l'extension que l'on accorde au mot "politique", seulement entre 1-1/2 et 5 pour cent de ses écrits ${ }^{1}$ seraient consacrés à la considération de ce genre de question. Et nous avons le témoignage de Kant lui-même pour nous indiquer le caractère peu systématique de certains de ses textes politiques les plus importants, notamment de son Projet de paix perpétuelle (1795), qui ne serait, au dire de Kant, que des "rêveries", une collection d"'opinions avancées au hasard". ${ }^{2}$ De plus, en ce qui concerne la partie plus systématique de son oeuvre politique, constituée surtout de la Doctrine du droit (1797), il a été suggéré, notamment par Friedrich Paulsen, que celle-ci aurait été rédigée pendant la période de sénilité du philosophe de Königsberg. ${ }^{3}$ A cet égard, effectivement, force nous est de reconnaître que la Doctrine du droit, ainsi que la Doctrine de la vertu, première et seconde parties, respectivement, de la Métaphysique des moeurs, présentent des difficultés de lecture considérables. La complexité et l'obscurité syntaxiques, allant parfois jusqu'à l'incorrection grammaticale, assorties d'une terminologie copieuse et, du moins en apparence, flottante, marquent très possiblement une détérioration par rapport aux trois grandes Critiques qui avaient précédemment fait la renommée de leur auteur et dont le style ne rendait déjà pas facile la tâche du lecteur. Nous ne retiendrons cependant pas l'hypothèse d'une période de sénilité pour rendre compte de l'impénétrabilité relative du texte de la Doctrine du droit, ni surtout pour servir de prétexte à notre paresse, car nous croyons que la détérioration du style de Kant, si détérioration il y a eu, a été voulue dans la mesure où elle est réelle et s'explique moins par l'âge avancé de l'auteur que par des changements survenus dans la situation politique à l'intérieur et à l'extérieur de la Prusse, ainsi que par la prudence qui s'imposait à un auteur qui traitait de questions politiques tout en habitant un pays à régime monarchique quatre ans à peine après la décapitation de Louis XVI. Il est d'ailleurs à remarquer que Kant avait déjà eu de sérieux démêlés avec la censure à propos de sa Religion dans les limites de la simple raison 
(1793) - et avait même fait l'objet d'une remontrance que lui adressait personnellement le roi Frédéric-Guillaume II. ${ }^{4}$

A Fichte, qui lui avait écrit en 1793 pour dire que son âme était "tout enflammée d'une merveilleuse idée"s qui lui permettrait de résoudre le problème proposé par Kant aux pages 372-373 de la deuxième édition de la Kritik der reinen Vernunft, Kant répond qu'il souhaite que Fichte le dévance dans cette affaire ou, plutôt, que ce dernier rende "dispensable" le rôle que Kant devait y jouer. ${ }^{6}$ Il s'agissait en l'occurrence de tracer les grandes lignes d'"une constitution ayant pour but la plus grande liberté humaine fondée sur des lois qui permettraient à la liberté de chacun de subsister en même temps que la liberté de tous les autres".' Philonenko rapporte que quelques mois plus tard Fichte écrit de nouveau à Kant pour lui annoncer "que son plan de travail intéressant le droit naturel, le droit politique (Staatsrecht) et la politique proprement dite est assez vaste pour occuper la moitié de sa vie sans préciser que l'autre moitié sera consacrée à fonder plus justement la philosophie transcendentale" ${ }^{8}$ Or ce n'est pas par pure générosité que Kant manifeste ce désir de s'effacer devant le jeune Fichte si plein d'enthousiasme. La même année dans une lettre à Spener où il est justement question des difficultés qu'il rencontre vis-à-vis la censure depuis la mort de Frédéric le Grand, Kant écrit que "lorsque les grands de ce monde sont dans un état d'intoxication, un pygmée qui chérit bien sa peau fait bien de ne pas s'impliquer dans leurs querelles". ${ }^{9}$ En décembre de la même année, Kant écrit à Kiesewetter qu'“en ce moment où la mode veut qu'on donne l'alarme là où il n'y a que tranquillité et paix, on doit donc patienter, tout en accordant une obéissance exacte à la loi". ${ }^{10}$ Plus de quatorze mois plus tard, en mars 1795 , en réponse à une requête que lui adressait Schiller de collaborer à un périodique (Die Horen - Les Heures) dont il était le rédacteur, Kant écrit qu'il doit "demander un assez long délai - um einen etwas langen Aufschub bitten". ${ }^{11}$ Kant poursuit en constatant que tout "matériel se rapportant à des questions d'état ou de religion se trouve pour le moment soumis à un certain blocus [Handelssperte], mais il n'y a guère en ce moment que cette sorte de marchandise qui intéresse la grande majorité des lecteurs". Kant ajoute que "l'on doit observer pendant encore quelque temps ce changement de climat, afin de s'accommoder prudemment du temps - um sich klüglich in die Zeit zu schicken". ${ }^{12}$ Quelques mois plus tôt Kant avait écrit à son éditeur Lagarde les lignes suivantes:

"Puisque mon sujet est à proprement parler la métaphysique dans l'acception la plus large du mot et s'occupe en tant que telle de théologie, de morale (et, avec cette dernière, de religion aussi), ainsi que de droit naturel (et, avec ce dernier, de droit politique et du droit des gens), mais seulement du point de vue de ce que la simple raison a à en dire, et que la main de la censure [ne] s'abat [pas moins] sur elle à présent, aucune garantie n'existe que l'ensemble du travail entrepris en ce domaine ne soit frustré par un trait de plume du censeur. - Lorsque la paix, qui semble être proche, s'établira, on peut espérer que les limites dans lesquelles un auteur doit se tenir seront plus exactement indiquées par des décrets plus précis, afin que celui-ci puisse se sentir en sécurité dans le domaine qui lui 
est laissé - so, dass er in dem, was ihm noch freigelassen wird, sich für gesichert halten kann. ${ }^{13}$

N'est-il pas significatif à cet égard, comme l'a fait remarquer Hettner, ${ }^{14}$ que Kant ait "publié ses écrits sous son propre nom, tandis que les Contributions à la juste appréciation de la Révolution française de Fichte paraissaient de façon anonyme"? Je pense que la conclusion qui s'impose ici est que dans notre lecture des écrits politiques de Kant nous ne devons ni ne pouvons faire abstraction de l'oeil du censeur qui, toujours présent à l'esprit de Kant, lisait, pour ainsi dire, par-dessus son épaule. ${ }^{15}$ Il ne serait pas surprenant, dès lors, s'il s'avérait que Kant se soit parfois exprimé par des tours de phrase qui n'auraient pas la même signification pour le lecteur averti que pour le fonctionnaire-théologien moyen. L'un des principes de base de notre lecture de la philosophie politique de Kant sera, en effet, qu'il faut examiner à la loupe pour en déterminer le sens précis, toute proposition qui semble, de prime abord, exprimer une position favorable au régime en place. Nous ne devons cependant pas croire que Kant ait fait imprimer quoi que ce soit qui aille à l'encontre de ses véritables convictions. ${ }^{16} \mathrm{~A}$ cela il aurait préféré le silence: "Rétracter et renier sa conviction intime, on peut lire dans les papiers laissés par Kant, est une bassesse - Widerruf und Verleugnung seiner inneren überzeugung ist niederträchtig - et nul ne doit en être supposé capable, mais le silence en un cas comme le présent, est le devoir d'un sujet; et si tout ce que l'on dit, doit être vrai, ce n'est cependant pas un devoir de dire publiquement toute vérité". ${ }^{17}$

Mais si Kant, dans ses écrits politiques, ne s'est pas exprimé avec toute la clarté voulue, et s'il s'avérait de plus qu'il n'ait pas tout dit sur sa véritable position, comment, dès lors, déterminer le sens des textes qu'il s'est néanmoins senti dans l'obligation de faire publier? Nous n'avons pas, il me semble, à cet égard, d'autre recours qu'à une lecture fouillée qui s'appuie sur l'aspect systématique de l'oeuvre de Kant. Or, malheureusement, au dire de Hans Saner, l'oeuvre politique de Kant a été délaissée précisément par ceux-là parmi ses commentateurs qui s'attachaient le plus aux aspects systématiques de sa pensée. ${ }^{18}$ D'autres commentateurs, aux préoccupations moins systématiques, ceux qui ne se sont pas laissés complètement rebuter par l'incohérence et l'intelligibilité apparentes du texte, ainsi que par certaines idées apparemment réactionnaires de l'auteur, se sont le plus souvent donné pour unique tâche de retrouver les origines des idées politiques de Kant. Et l'on y a si bien réussi, comme le dit encore Hans Saner, que les nombreux pères $^{19}$ des idées politiques de Kant ont été retrouvés, mais Kant lui-même a été perdu. Car si l'on a tant cherché à situer la position de Kant par rapport à la pensée des autres, c'est souvent qu'on arrivait mal à la situer par rapport au système kantien dans son ensemble, et qu'on évitait ainsi d'avoir à la confronter en tant que philosophie politique. Mais déjà, heureusement, un mouvement contraire s'esquisse. Selon Eric Weil, "Kant, ici comme partout, marque un tournant dans l'histoire de la philosophie", et ce justement en raison des préoccupations systématiques du philosophe de Königsberg, Chez Kant, en effet, selon l'interprétation de Weil, "la politique devient problème parce que la morale et, mais seulement à sa suite, la philosophie de la nature 
ne permettent plus d'éviter cette question: ni l'une ni l'autre ne seraient achevées, ni l'une ni l'autre ne seraient même vraies, si elles ne donnaient pas de réponse à ce problème qu'elles posent et imposent au philosophe. Ce n'est pas la réflexion politique qui détermine la philosophie kantienne, c'est cette philosophie qui conduit, non aux problèmes politiques, mais au problème de la politique". ${ }^{20}$ Les limitations d'espace ne permettent toutefois pas dans le cadre du présent essai de faire le lien entre la pensée politique de Kant et tout le système des Critiques, bien que ceci ait déjà été tenté, notamment par Hans Saner. Nous entendons cependant, au cours des remarques qui vont suivre, faire état au moins de la cohérence interne de la philosophie politique kantienne et de ses rapports avec la philosophie morale, en plus de nous servir de cette cohérence comme principe d'interprétation de certains points obscurs ou controversés.

S'il y a pour Kant un problème central en philosophie politique, autour duquel tojustification de la contrainte juridique, qui constitue d'ailleurs la condition récessaire de l'existence même d'un Etat politique et d'un système de lois garantissant la liberté. Mais, en effet, on ne peut éviter de se poser la question: cette contrainte peut-elle se justifier moralement? Et, si oui, comment et dans quelles conditions? La thèse des anarchistes, qui ne tardera pas à voir la lumière du jour, n'est-elle justement pas que, sauf en cas de légitime défense, la contrainte est toujours et partout une agression indue, une violence perpétrée contre des individus et des groupes, peu importe qu'elle procède du caprice sauvage d'un autre individu ou qu'elle procède de l'Etat et qu'elle s'exerce au nom de la collectivité selon des lois. La question du droit et, partant, de la politique est donc celle de la justification de la contrainte. "Ainsi, dit Kant, le droit et l'autorisation [Befugnis] de contraindre sont une seule et même chose". ${ }^{21}$

Or, malgré tout ce qu'on a pu dire pour faire de Kant l'ancêtre du positivisme juridique moderne, ${ }^{22}$ l'autorisation dont il s'agit dans ce passage est bien une autorisation morale, et la morale en l'occurrence est bien la morale kantienne. Dans la Doctrine de la vertu, Kant dit très clairement que "le devoir de vertu se distingue essentiellement du devoir de droit en ce qu'une contrainte extérieure est moralement possible par rapport à ce dernier, tandis que le premier repose uniquement sur une libre contrainte personnelle". ${ }^{23}$ Dans l'Introduction générale à la Métaphysique des moeurs, Kant explique que les "lois de la liberté sont appelées morales à la différence des lois de la nature. Lorsqu'elles ne portent que sur des actions extérieures et leur légalité, elles sont dites juridiques; mais si, de plus, elles exigent d'être en tant que telles (comme lois) les principes de détermination des actions, elles sont alors éthiques" (Doctrine du droit, p. 88). Il est donc clair que les devoirs de droit constituent dans l'idée de Kant un sous-ensemble par rapport aux devoirs éthiques.

Sournoisement, par ces définitions, Kant vient de refuser d'accorder un statut proprement juridique à toute mesure prévoyant une contrainte qui soit contraire à la morale! (Ainsi, nous pouvons imaginer que Kant ne dirait pas d'une prétendue loi obligeant chaque citoyen à retourner au propriétaire tout esclave évadé qui se réfugierait chez lui qu'elle est injuste; il dirait bien plutôt que ce n'est même pas une loi, puisqu'aucune autorisation morale n'existe 
pour justifier une telle contrainte, qui ne serait autre chose que violence injustifiée; on obéit à de telles "lois" par prudence seulement, lorsque, effectivement, on y obéit, comme on obéirait à une bande de brigands armés, faute de moyens de résistance mais sans accorder la moindre légitimité à leurs demandes.) Kant s'exprime, il est vrai, un peu moins clairement sur ce point au début du premier chapitre de la Doctrine du droit où il s'agit d'expliquer en quoi consiste la "doctrine du droit" - mais il ne faut pas oublier que ce passage, par sa situation au début du premier chapitre ainsi que par le sujet qu'il se donne, serait l'un de ceux sur lesquels le censeur exercerait sa plus grande vigilance. Kant y dit que la "doctrine du droit" est "la totalité des lois pour lesquelles une législation extérieure est possible", ${ }^{24}$ en omettant, cette fois, de préciser que la possibilité dont il est question ici est bien une possibilité morale et non pas, par exemple, une simple possibilité technique, ou même logique. Kant ajoute que lorsqu'une telle législation existe dans les faits, c'est-à-dire lorsqu'une législation qui est moralement possible existe dans les faits, il s'agit alors de la doctrine du droit positif. Kant avait précédemment expliqué que les lois positives sont celles "qui sans une législation extérieure réelle n'obligeraient pas et ne seraient pas des lois" (Doctrine du droit, p. 99). Un exemple d'une loi positive serait donc la loi des provinces canadiennes qui oblige les automobilistes à conduire du côté droit, ou la loi britannique qui oblige à conduire du côté gauche du chemin. Ces lois, en plus de faire peser sur l'automobiliste une certaine contrainte exercée par la police et les tribunaux, créent une certaine obligation morale de s'y conformer, obligation qui n'existerait pas en l'absence d'une telle législation, comme dans les pays où le côté du chemin duquel on doit se tenir ne fait l'objet d'aucune loi.

L'obligation qui s'attache à ce que Kant appelle une loi naturelle, par contre, "peut être reconnue a priori par la raison, en l'absence même de législation extérieure". ${ }^{25}$ Un exemple d'une loi naturelle serait donc celle qui interdit l'homicide volontaire sauf en certains cas de légitime défense, car la raison reconnaît d'emblée que je ne saurais accepter qu'un autre choisisse de me tuer dans le but de se procurer quelque avantage. Il est à remarquer que, selon cette définition de Kant, une loi ne cesse pas pour autant d'être "naturelle" lorsque l'homme sort de l'état de nature et prend des mesures pour la faire respecter par tous et chacun. Il s'ensuit que ce que l'on appelle aujourd'hui des "lois positives", dans l'acception courante du terme, comprendrait, entre autres, des lois naturelles au sens de Kant, lorsque celles-ci seraient l'objet d'une législation extérieure effective. Notre terme "loi positive" comprendrait aussi des lois positives au sens de Kant, ainsi que des mesures gouvernementales auxquelles Kant refuserait jusqu'au titre de "lois".

Kant se demande si une législation extérieure composée uniquement de lois positives, est possible - et répond dans l'affirmative, avec la qualification très importante qu'il faudrait "qu'une loi naturelle précédât pour fonder l'autorité du législateur (c'est-à-dire l'autorisation [Befugnis] de lier [les] autres par son simple arbitre [durch seine blosse Willkür]". On dirait qu'il s'agit ici d'une distinction pointilleuse, sans beaucoup d'importance, et c'est précisément l'impression que Kant aurait voulu créer dans l'esprit du 
fonctionnaire-théologien moyen qui assure la fonction de censeur dans l'Etat prussien. Celui-ci n'aurait-il pas tendance à prendre les termes de "lois positives" et de "lois naturelles" dans leurs acceptions habituelles? Ainsi il retiendrait surtout que dans un système politique donné il pourrait n'y avoir que des lois positives, au sens habituel du mot. Nous n'avions évidemment pas besoin de Kant pour nous le dire; mais, penserait notre fonctionnaire, ces vieux professeurs se plaisent à dire des vérités évidentes et, au fond, insignifiantes, en donnant l'impression d'avoir fait une grande découverte et en s'exprimant d'une façon délibérément obscure! La seule "loi naturelle" qui compte, dans l'Etat prussien par exemple, serait celle qui fonde l'autorité du législateur - et ici notre fonctionnaire penserait à l'autorité du roi, son "souverain" - qui contraint ses sujets "par son simple arbitre". Mais, à la vérité, dans ce passage, au contraire de la lecture qu'en ferait notre fonctionnaire, Kant rappelle au lecteur attentif et averti le virement de sens qu'il a effectué par rapport au terme "loi positive": dans l'acception où Kant emploie désormais ce terme il n'y a plus de loi, et donc plus de loi positive, dès qu'il n'est plus possible de justifier la coercition qu'elle comporte par le recours à une construction (ou à une reconstruction) rationnelle, ce qui signifie, comme nous verrons plus loin, une dérivation de l'impératif catégorique - ou, plus précisément, une dérivation de l'autorisation de contraindre contenue implicitement dans l'impératif catégorique.

Ce qui est essentiellement le même point revient lorsque Kant tente de lever l'ambiguïté qu'il constate dans l'emploi du terme "état de nature" qui, effectivement, sert tantôt à désigner l'état où l'homme se trouverait en l'absence complète d'influences sociales, tantôt à désigner la situation qui existerait en l'absence de liens proprement juridiques entre les hommes. Ainsi Rousseau, dont Kant ne nous parle pas ici mais dont la présence se devine toujours à l'arrière-plan, ne décrit-il pas dans son Discours sur l'origine et les fondements de l'inégalité entre les hommes (1755) l'homme "tel qu'il a dû sortir des mains de la nature" ? ${ }^{26}$ C'est donc l'homme, abstraction faite de tout ce que la société a pu faire de lui, qui préoccupe Rousseau. Ici, la liberté naturelle de l'état de nature est celle qui précède toutes les contraintes que la société fait peser sur l'individu et toutes les autres influences qu'elle exerce sur lui. Mais dans le Contrat social, Rousseau nous parle d'un état de nature tout autre, qui surgit dans une société déjà très évoluée par rapport à ce que connaissait l'homme vivant solitaire au fond de la forêt du Discours sur l'inégalité. Cet état de nature n'apparaît, effectivement, que lorsque les liens juridiques entre les hommes sont dissous par la rupture du pacte social: "A l'instant où le gouvernement usurpe la souveraineté [qui, selon Rousseau, n'appartient en propre qu'au corps politique constitué de tous les citoyens], ${ }^{27}$ le pacte social est rompu; et tous les simples citoyens, rentrés de droit dans leur liberté naturelle, sont forcés, mais non pas obligés d'obéir". ${ }^{28}$ L'homme qui se trouve dans l'état de nature, pris dans ce deuxième sens du terme, n'est souvent libre qu'au point de vue moral; en réalité, il se trouve, selon le mot de Rousseau, "dans les fers". ${ }^{29}$ Et il ne retrouvera sa liberté que lorsque tous auront contracté avec tous de n'obéir qu'aux lois consenties par ce que Rousseau appelle la volonté générale. De façon semblable, Kant écrit: 
"L'état juridique est ce rapport des hommes entre eux, qui contient les conditions sous lesquelles seules chacun peut jouir de son droit, et le principe formel de la possibilité de cet état, considéré d'après l'Idée d'une volonté législatrice universelle, est la justice publique [qui, selon Kant, comprend nécessairement ce qu'il appelle la "justice distributive"] . . .

L'état non juridique, [par contre,] c'est-à-dire celui où il n'y a pas de justice distributive, s'appelle état de nature (status naturalis). Le contraire de celui-ci serait non pas (comme le pense Achenwall) l'état social, qui pourrait s'appeler un état artificiel (status artificialis), mais plutôt l'état civil d'une société soumise à une justice distributive, car dans l'état de nature aussi il peut exister des sociétés légitimes [rechtmässige Gesellschaften $^{30}$ ] (par exemple [la société] conjugale, [la société] paternelle, [la société] domestique en général, et bien d'autres ${ }^{31}$ ), pour lesquelles aucune loi a priori ne signifie: "Tu dois entrer en cet état", comme on peut dire, en effet, de l'état juridique (vom rechtlichen Zustande) [, à savoir qu'une loi a priori exige] que tous les hommes qui peuvent s'engager les uns par rapport aux autres (et ce même contre leur gré) dans des rapports juridiques, doivent entrer en cet état.» ${ }^{32}$

On peut se demander quelles sociétés précisément Kant avait en tête en parlant des nombreuses "autres" sociétés qui, dans l'état de nature, sont rechtmässig, sans pour autant être rechtlich. Quel serait, par exemple, à cet égard, le statut de la monarchie prussienne? Quoi qu'il en soit, il ressort clairement ici que contrairement à ce que suggèrent bien des interprétations de la philosophie politique de Kant, il ne suffit pas que je me trouve dans des rapports sociaux avec d'autres et soumis à des règlements contraignants pour que l'on puisse dire que je dois un devoir d'obéissance absolue à ces règlements comme s'il s'agissait d'un état proprement juridique. Pour Kant, comme pour Rousseau, il y a des cas où l'on choisit d'obéir (comme à son père après avoir atteint l'âge de raison), mais sans qu'il y ait obligation morale d'obéir; il existe selon les deux auteurs d'autres cas encore où l'on est forcé d'obéir, et où l'on obéit par prudence seulement. L'obéissance que nous devons à de vraies lois positives (et naturelles), nous ne la devons qu'à des mesures qui font l'objet d'une législation extérieure sous un régime proprement juridique (et donc en conformité avec la morale).

Mais qu'est-ce qu'un régime ou un Etat proprement juridique selon Kant? Et comment peut-il faire valoir qu'il existe un devoir moral d'entrer en cet état? Revenons donc un peu en arrière pour nous rappeler brièvement les grandes lignes de la théorie kantienne des fondements de la morale. La morale, chez Kant comme chez d'autres théoriciens, se rapporte essentiellement aux actions humaines. Une action, au sens propre du terme, comporte une certaine conscience (du moins en puissance) d'avoir recours à certains moyens (comme par exemple à un certain mouvement de mon bras et de ma main) afin d'accomplir un but que l'on se donne (comme par exemple celui de saisir une pomme que l'on a envie de manger). Les mouvements proprement involontaires, par contre, ne comportent pas de telle conscience (pas même en puissance). Les actions proprement dites se distinquent de ceux-ci par le fait qu'en ce qui les concerne le rapport moyen/fin est toujours susceptible de 
prendre la forme de ce que Kant appelle une "maxime" ou "principe subjectif d'action": J'étends ma main, par exemple, afin de prendre une pomme; je prends la pomme pour la manger; je la mange afin d'assouvir ma faim. Et, de plus, selon Kant du moins, c'est par la considération de la maxime de l'action que l'on peut déterminer si l'action en question est moralement permise ou moralement défendue. Le contrôle se fait de la manière suivante: celui qui agit doit se demander si, étant donné le but qu'il cherche à atteindre par son action, il pourrait accepter (ou vouloir) que d'autres personnes tâchent d'atteindre pour elles-mêmes un même but par des moyens semblables à celui qu'il envisage d'employer. $\mathrm{Si}$, par exemple, afin de répondre à mon désir de me rendre dans une ville voisine, je force le conducteur d'une automobile à me la céder à bout de fusil pour ensuite m'en servir, j'aurais commis par là une action qui, selon Kant, est moralement défendue. Car je ne saurais vouloir (ou même accepter) que la maxime de mon action ("je prends cette voiture par la force afin de me rendre là où je veux aller") soit adoptée par d'autres que moi, puisque quelqu'un d'autre, en adoptant une telle maxime, pourrait me ravir la voiture même dont je viens de prendre possession, me frustrant ainsi dans l'accomplissement de mon but. Précisons qu'il s'agit ici du point de vue rationnel quant à ce que je peux ou non vouloir: en adepte du Zen ou de la résignation chrétienne, je peux évidemment accepter tout ce qui m'arrive. Il n'est cependant pas rationnel, par le même mouvement de l'esprit par lequel je me donne un but, d'accepter ni surtout de vouloir qu'une autre personne agisse précisément d'une façon qui aura pour effet d'empêcher que je réalise le but en question. Voilà le sens de ce que Kant appelle l'impératif catégorique: "Agis seulement d'après une maxime [telle] que tu peux vouloir ... qu'elle devienne une loi universelle [, c'est-à- dire qu'elle serve de principe d'action à n'importe qui ayant des buts semblables aux tiens]". ${ }^{33}$

Dans le contexte de la philosophie politique et juridique, il s'agit donc de se demander si la maxime qu'on se donnerait en établissant une législation extérieure contraignante peut être celle d'une action qui soit moralement permise. Kant écrit:

«La résistance opposée à ce qui fait obstacle à un effet fait avancer celuici et s'accorde avec lui. Or, tout ce qui est injuste est un obstacle à la liberté suivant des lois universelles; mais la contrainte est un obstacle ou une résistance exercée sur la liberté. Il s'ensuit que si un certain usage de la liberté même est un obstacle à la liberté suivant des règles universelles (c'est-à-dire est injuste), alors la contrainte qui lui est opposée, en tant qu'obstacle à ce qui fait obstacle à la liberté, s'accorde avec cette dernière suivant des lois universelles, c'est-à-dire qu'elle est juste; par conséquent une autorisation (Befugnis) de contraindre ce qui lui est préjudiciable est, suivant la loi de contradiction, liée . . . au droit.» ${ }^{34}$

Voilà pour la preuve que dans certaines circonstances du moins il peut être moralement permis, au nom même de la plus grande liberté de tous selon des principes universels, d'imposer par moyen de la contrainte extérieure des limites à l'exercice de la liberté personnelle. Mais ce n'est pas n'importe quelle contrainte qui a pour effet d'augmenter la liberté de tous selon des principes universels. Pour préciser les conditions et les modalités de l'application d'une 
telle contrainte un plus long développement sera nécessaire. Mais Kant en dégage néanmoins tout de suite le principe de base: le droit, selon lui, "ne peut pas être pensé comme constitué de deux moments: à savoir de l'obligation suivant une loi - et de l'autorisation [Befugnis] de celui qui oblige les autres par son arbitre de les contraindre à accomplir cette obligation". ${ }^{35} \mathrm{Au}$ contraire, le droit strict exigerait "une contrainte réciproque complète", comme dans la doctrine de la volonté générale de Rousseau ${ }^{36}$ Chez Kant, en effet, comme chez Rousseau, "le pouvoir législatif ne peut appartenir qu'à la volonté unifiée du peuple" (Doctrine du droit, p. 196). Les membres d'une société vivant sous une condition proprement juridique y retrouvent "la liberté légale de n'obéir à aucune autre loi qu'à celle à laquelle ils ont donné leur consentement". Ainsi, le souverain, "considéré selon les lois de la liberté, ne peut être autre que le peuple uni luimême" (Doctrine du droit, p. 198).

Il reste que le souverain, chez Kant comme chez Rousseau, ne statue que sur les conditions générales des rapports qui existeront entre les citoyens et ne peut pas, par la force des choses, s'occuper de l'application des lois à des cas particuliers. Le souverain ne peut donc ni assumer les fonctions d'un tribunal ni exercer celles du gouvernement proprement dit. Kant écrit:

«Le régent de l'Etat (rex, princeps) est la personne (morale ou physique) investie du pouvoir exécutif . . .: c'est l' agent de l'Etat, qui installe les magistrats, prescrit au peuple les règles d'après lesquelles conformément à la loi ( par subsomption d'un cas sous cette loi), chacun peut . . . acquérir ou conserver ce qui est sien. . . . Les ordres qu'il donne au peuple . . . sont des ordonnances, des décrets (non des lois), car ils ont pour objet une décision en un cas particulier et ils se donnent comme révocables. Un gouvernement, qui serait en même temps législateur, devrait être appelé despotique. . . ." (Doctrine du droit, p. 199)

Inversement, si le régent n'a pas l'autorisation de légiférer, le législateur ou souverain ne peut pas gouverner à la place du régent:

«Le souverain [Beherrscher] du peuple (le législateur) ne peut ainsi être en même temps le régent; car ce dernier est soumis à la loi et est donc obligé par celle-ci, et donc obligé par un autre (le souverain). Le souverain peut lui ôter son pouvoir, le déposer, transformer son administration, mais il ne peut le punir . . .; car il s'agirait là encore d'un acte du pouvoir exécutif, auquel revient, suivant la loi, la faculté [Vermögen] de contraindre d'une manière suprême, qui néanmoins serait soumis à une contrainte; en quoi il y a contradiction.» (Doctrine du droit, p. 199)

C'est d'ailleurs en raison de cette dernière déclaration et d'autres semblables qu'on a si souvent reproché à Kant d'avoir nié le droit de résistance aux autorités établies, même en cas d'abus grave des pouvoirs du gouvernement. Et, effectivement, il est exact de prétendre que la résistance armée n'est nullement, selon Kant, un moyen d'action juridique. On ne saurait, par exemple, inscrire dans la constitution même d'un Etat juridique quelque droit que ce soit de s'opposer par la force au gouvernement. Cette idée, que 
l'on retrouve chez Achenwall, Fichte et les auteurs des premières constitutions française et américaine, serait, selon Kant, contradictoire. Toute révolution constitue pour lui, en effet, une destruction du système juridique en place. Mais ce que Kant ne dit pas ici d'un ton trop haut, c'est qu'il se pourrait que le régent se soit déjà conduit d'une façon qui détruise le caractère juridique de la contrainte qu'il fait subir au peuple. Cette contrainte ne s'exerçant plus conformément à la loi, le peuple se retrouverait replongé dans un état de nature par rapport à son ancient régent, contre qui le peuple aurait le même droit de légitime défense que contre n'importe quel autre agresseur. Le devoir de contraindre celui qui se maintient par rapport à nous dans un état de nature, à entrer avec nous dans un état juridique s'appliquerait d'ailleurs tout autant dans le cas d'un ancien régent que dans le cas de n'importe quel autre individu. Là où il existe un véritable Etat juridique, certes, le régent est irrésistible; mais si l'on réussit à déposer par la force celui qui se donnait pour régent, on démontre seulement que ce dernier n'était pas le régent d'un Etat juridique, puisque la violence sauvage a eu raison de lui. Il lui manquait le pouvoir unique et suprême de contraindre selon des lois, condition sine qua non de l'Etat juridique. Voilà le véritable sens de la formule de Kant selon laquelle dans un Etat juridique le pouvoir exécutif est irrésistible.

\section{James Crombie \\ Université Ste-Anne}

\section{Notes}

' D'après l'estimé de Hans Saner, Kant's Political Thought: Its Origins and Development, trad. de l'allemand par E.P. Ashton (Chicago/Londres, 1973), p. 2.

2 Lettre à Kieswetter (1795), citée par Saner, ibid.

${ }^{3}$ Cf. Friedrich Paulsen, Immanuel Kant, sein Leben und sein Lehre, $5^{0}$ éd. (Stuttgart, sans date), p. 364; cité par Saner, op. cit., p. 316, note 16: "L'exposition systématique des fondements métaphysiques de la Doctrine du droit (1797) appartient à la période de sénilité. . . . Ceci est vrai également du deuxième essai, qui fait partie du Conflit des facultés (1798)". Tel n'est cependant pas l'avis de J. Gibelin, qui, dans son "Introduction du traducteur" au Conflit des facultés de Kant (Paris, 1973), pp. iv-v, répond à l'affirmation de K. Fischer selon laquelle Kant "savait que dans ses exposés oraux et écrits se manifestaient déjà visiblement les traces d'une intelligence décroissante. . ." par ce qui suit: "En ce qui concerne le Conflit des facultés, cette opinion nous paraît exagérée, sinon erronée. La différence entre les trois parties du traité a été, comme on le verra, voulue par Kant et s'explique aisément si l'on tient compte des circonstances, de l'ambiance intellectuelle et des principes mêmes de la philosophie de l'auteur."

${ }^{4}$ Cf. Theordore M. Greene, "The Historical Context and Religious Significance of Kant's Religion", dans Kant, Religion within the Limits of Reason Alone, trad. de l'allemand par Greene et John R. Silber (New York, 1960), esp. pp. xxxii-xxxvi.

5 "Dans glüht meine Seele von einem grossen Gedanken. . .", cité par A. Philonenko, Théorie et praxis dans la pensée morale et politique de Kant et de Fichte en 1793 (Paris, 1968), p. 80

6 Lettre à Fichte (1793), citée par Saner, op. cit., p. 2.

7 Critique de la raison pure, trad. par A. Tramesaygues el B. Pacaud, (Paris, 1965), p. 264.

8 Philonenko, p. 80.

9 Lettre à Spener (1793), citée par Saner, p. 2.

10 Lettre à Kiesewetter (1793) citée par Werner Haensel, Kants Lehre vom Widerstandsrecht: Ein Beitrag zur Systematik der Kantischen Rechtsphilosophie (Berlin, 1926), p. 76. Haensel, cependant, cherche à démontrer que ni la position de Kant en ce qui concerne l'autorité de l'Etat ni sa façon de la présenter n'ont été déterminées par l'“Aufluss von Zensurrücksichten”. 
Nous sommes pleinement d'accord avec Haensel sur le premier point, mais en désaccord sur le deuxième.

1 Lettre à Schiller (1974), citée par Saner, p. 77.

12 Cf. ibid.: ". . . ausser diesen [Materialen] kaum noch, wenigstens in diesem Zeitpunkt, andere die grosse Lesewelt interessierende Artikel gibt. . . ."

13 Lettre à Lagarde (1794), citée par Saner, p. 76.

14 Literaturgeschichte des 18. Jahrhunderts (Braunschweig, 1879), p. 46; cité par Haensel, p. 74.

15 Pour une bibliographie sommaire de cette question du "Conflit de la censure", voir Georges Vlachos, La Pensée politique de Kant (Paris, 1962), p. 541, note 76.

${ }^{16}$ Suivant Friedländer, "Kant in seiner Stellung zur Politik", Deutsche Rundschau, 9 (1876), 241 et sqq.; cité par Haensel, pp. 74 et 77.

17 2e "Pièce annexe" au Conflit des facultés, trad. Gibelin, p. 143; citée en raccourci par Haensel, p. 77.

${ }_{18}$ Voir Saner, p. 3; dans la note qui intéresse la remarque en question ( $\mathrm{n}^{\circ} 18$ à la p. 316), Saner poursuit en donnant le nombre de pages consacrées aux théories politiques de Kant dans les ouvrages suivants, respectivement: J.E. Erdmann, Der Entwicklung der deutschen Spekulation seit Kant, vol. I (Stuttgart, 1931) [8 sur 232 pages]; Paulsen [20 sur 420 pages]; Bruno Brauch, Immanuel Kant (Berlin/Leipzig, 1971) [13 sur 470 pages]; Ernst Cassirer, Kants Leben und Lehre (Berlin, 1918) [15 sur 460 pages]. La seule exception à cette règle, selon Saner, serait Karl Jaspers qui, pour la "première fois", accorderait aux idées politiques de Kant le "rang métaphysique" qui leur revient.

19 Dans la liste de ceux à qui on a déjà attribué une telle paternité, Saner mentionne (note 17, p. 316) les noms de Machiavel, Hobbes, Locke, Hume, Grotius, Pufendorf, Thomasius, Leibniz, Wolff, Baumgarten, Vattel, Achenwall, Fichte, Humboldt, Gentz, Montesquieu, Rousseau, Voltaire, Condorcet, d'Argenson, Turgot, Mirabeau, de Sieyès, et "d'autres encore".

${ }^{20}$ Eric Weil, "Kant et le problème de la politique", dans La Philosophie politique de Kant (Paris, 1962), pp. 1,3.

${ }^{21}$ Métaphysique des moeurs, première partie: Doctrine du droit, trad. par A. Philonenko (Paris, 1971), p. 106. D'autres références à cette oeuvre seront signalées dans le texte. Nous n'avons pas cependant retenu la traduction du terme "Befugnis" par "faculté" adoptée par Philonenko: "Recht und Befugnis zu zwingen bedeuten also einerlei." - Die Metaphysik der Sitten, Erster Theil: Anfangsgründe der Rechtslehre, dans Kants Werke: Akademie Textausgabe, vol. VI (Berlin, 1968), p. 232.

22 Voir à cet égard, Michel Villey, "Kant dans l'histoire du droit", dans la Philosophie politique de Kant, pp. 53-76.

${ }^{23}$ Métaphysique des moeurs, deuxième partie: Doctrine de la vertu, trad. par A. Philonenko (Paris, 1968), p. 52; cf. Die Metaphysik der Sitten, Zweiter Theil: Metaphysische Anfangsgründe der Tugendlehre, dans Kants Werke, VI, p. 383.

${ }^{24}$ Rechtslehre, p. 229; cf. Doctrine du droit, p. 103.

${ }^{25}$ Rechtslehre, p. 224.

26 Oeuvres complètes, présentation par Michel Launay (Paris, 1971), II, p. 213.

27 Cf. Livre I, ch. vi; loc. cit., pp. 522-523.

28 Livre III, ch. x, p. 554.

29 Livre I, ch. i, p. 518.

${ }^{30}$ Comment traduire "rechtmässig"? Le Dictionnaire de poche des langues française et allemande de Langenscheidt (Berlin, 1940), 2e partie, p. 305, suggère les trois traductions suivantes: "légal . . ." légitime . . .; juste".

${ }^{31}$ C'est moi qui souligne ces quatre petits mots, possiblement lourds de signification. Voir infra.

${ }^{32}$ Rechtslehre, pp. 305-306; cf. Doctrine du droit, pp. 187-188.

${ }^{33}$ Ibid., p. 225; cf. Doctrine du droit, p. 99. Il s'agit d'une version améliorée de la formulation de 1785 (cf. Grundlegung zur Metaphysik der Sitten, dans Kants Werke, vol. IV, p. 421). C'est le principe fondamental de nos devoirs éthiques. Ces derniers sont soit de vertu, soit de droit. Pour le principe fondamental de chacune de ces catégories de devoir, cf. Doctrine de la vertu, p. 67 (Tugendlehre, p. 395) et Doctrine du droit, p. 105 (Rechtslehre, p. 225), respectivement.

${ }^{34}$ Rechtslehre, p. 231; cf. Doctrine du droit, pp. 105-106. H. Cohen, Kants Begründung der Ethik, insiste à juste titre que chez Kant la contrainte est "conditionnée" par la liberté. Haensel, notamment, (op. cit.) attribue aux "tendances socialistes" de Cohen, ce qu'il qualifie 
de certaines erreurs d'interprétation de la pensée politique de Kant.

${ }^{35}$ Ibid., pp. 232/106.

${ }^{36}$ Cf. Contrat social, I, i, pp. 522-524. 